\title{
Selective Immunolesions of Cholinergic Neurons in Mice: Effects on Neuroanatomy, Neurochemistry, and Behavior
}

\author{
Joanne Berger-Sweeney, ${ }^{1}$ Nancy A. Stearns, ${ }^{1}$ Stephanie L. Murg, ${ }^{2}$ Laura R. Floerke-Nashner, ${ }^{1}$ \\ Douglas A. Lappi, ${ }^{3}$ and Mark G. Baxter ${ }^{2}$ \\ ${ }^{1}$ Department of Biological Sciences, Wellesley College, Wellesley, Massachusetts 02481, ${ }^{2}$ Department of Psychology, \\ Harvard University, Cambridge, Massachusetts 02138, and ${ }^{3}$ Advanced Targeting Systems, San Diego, California 92121
}

The ability to selectively lesion mouse basal forebrain cholinergic neurons would permit experimental examination of interactions between cholinergic functional loss and genetic factors associated with neurodegenerative disease. We developed a selective toxin for mouse basal forebrain cholinergic neurons by conjugating saporin (SAP), a ribosome-inactivating protein, to a rat monoclonal antibody against the mouse p75 nerve growth factor (NGF) receptor (anti-murine-p75). The toxin proved effective and selective in vitro and in vivo. Intracerebroventricular injections of anti-murine-p75-SAP produced a dose-dependent loss of choline acetyltransferase (ChAT) activity in the hippocampus and neocortex without affecting glutamic acid decarboxylase (GAD) activity. Hippocampal ChAT depletions induced by the immunotoxin were consistently greater than neocortical depletions. Immunohistochemical analysis revealed a dose-dependent loss of cholinergic neurons in the medial septum (MS) but no marked loss of cholinergic neurons in the nucleus basalis magnocellularis after intracerebroventricular injection of the toxin. No loss of noncholinergic neurons in the MS was apparent, nor could we detect loss of noncholinergic cerebellar Purkinje cells, which also express p75. Behavioral analysis suggested a spatial learning deficit in anti-murine-p75SAP-lesioned mice, based on a correlation between a loss of hippocampal ChAT activity and impairment in Morris water maze performance. Our results indicate that we have developed a specific cholinergic immunotoxin for mice. They also suggest possible functional differences in the mouse and rat cholinergic systems, which may be of particular significance in attempts to develop animal models of human diseases, such as Alzheimer's disease, which are associated with impaired cholinergic function.

Key words: Alzheimer's disease; basal forebrain; cholinergic; immunotoxin; saporin; p75
Immunotoxins are powerful, cost-effective tools that are used to make selective neurochemical lesions, investigate specific functions of defined neural systems, and model human disease states. Immunotoxins are composed of a cytotoxin bound to an antibody directed against a cell-surface protein specific to a particular cell population. In particular, the immunotoxin 192-saporin (SAP), directed against the rat p75 low-affinity nerve growth factor (NGF) receptor, has proved extraordinarily useful in investigating the functions of the basal forebrain cholinergic system in rats (Wiley, 1992; Wrenn and Wiley, 1998; Baxter and Chiba, 1999).

Because of a growing interest in studying neurobiology in mouse models combined with the ease of genetic manipulation in this species, it is of great interest to develop a toxin similar to 192-SAP that is effective in mice. There are a number of transgenic mouse models of Alzheimer's disease (AD) that reproduce particular aspects of AD pathology, including amyloid deposits, plaque and tangle formation, and neurodegeneration (for review, see Bornemann and Staufenbiel, 2000; Janus et al., 2000; Price et

Received June 5, 2001; revised July 18, 2001; accepted July 26, 2001.

This work was supported in part by a Brachman-Hoffman grant. We thank Jing-Yu Pan for assistance with the behavioral experiments, Carol Ann Paul for help in establishing the immunocytochemical procedures for the confocal microscope, and Drs. Urs Berger and Karyn Frick for their work on earlier versions of this toxin.

Portions of the research reported in this article have appeared previously in abstract form (Berger-Sweeney et al., 2000).

Correspondence should be addressed to Dr. Joanne Berger-Sweeney, Department of Biological Sciences, 106 Central Street, Wellesley College, Wellesley, MA 02481. E-mail: jbergers@wellesley.edu.

Copyright (ㄷ) 2001 Society for Neuroscience $\quad 0270-6474 / 01 / 218164-10 \$ 15.00 / 0$ al., 2000). Although loss of basal forebrain cholinergic neurons may not be the initial neurodegenerative event in $\mathrm{AD}$, it is thought that cholinergic loss plays a role in cognitive impairment in $\mathrm{AD}$ and might exacerbate the functional impairment and neurodegeneration in AD (for review, see Kasa et al., 1997; Wenk and Willard, 1998; Whitehouse, 1998). Therefore, the ability to selectively lesion basal forebrain cholinergic neurons in experimental mouse models of AD may significantly enhance the utility of these models in understanding the pathogenesis of neurodegeneration and cognitive impairments in AD.

The 192-SAP immunotoxin is effective in the rat but does not bind to mouse cells. Therefore, to develop a specific cholinergic immunotoxin for mice, we conjugated a rat monoclonal antibody against the mouse p75 receptor (anti-murine-p75) to SAP. The anti-murine-p75-SAP was tested in vitro for efficacy (experiment 1). We then performed in vivo dose-response experiments in mice to establish the efficacy of the toxin in the intact mouse (experiment 2). After selection of optimal doses of toxin, we tested mice with intracerebroventricular toxin injections on a spatial learning task, a $1 \mathrm{~d}$ version of the Morris water maze, as a preliminary assessment of the behavioral effects of cholinergic depletion in the mouse brain (experiment 3).

\section{Experiment 1}

This experiment was designed to assess the in vitro effectiveness of the immunotoxin in killing cells that express murine p75. The in vitro toxicity of different doses of anti-murine-p75-SAP was assessed in cultured NG3 cells that express both murine and rat p75. 


\section{Experiment 2}

After in vitro testing, we examined the effectiveness of the toxin in vivo. Mice received either saline or one of several different doses of immunotoxin injections into the lateral ventricles (intracerebroventricularly). At 2-4 weeks after surgery, the hippocampi and somatosensory cortices of the lesioned mice were assessed for choline acetyltransferase (ChAT) activity to examine the efficacy of the toxin in reducing cholinergic activity in basal forebrain targets. Glutamic acid decarboxylase (GAD) activity was measured in the same target regions to assess nonspecific damage after different doses of the toxin. In other lesioned mice, ChAT immunostaining was performed to assess the efficacy of the toxin in killing cholinergic basal forebrain neurons in the medial septum (MS) and nucleus basalis magnocellularis (nBM). In alternate sections, parvalbumin and calbindin immunostaining was performed to assess the specificity of the toxin, because those markers are expressed in noncholinergic basal forebrain neurons in the MS and nBM (Freund, 1989; Heckers et al., 1994).

\section{Experiment 3}

Once we had determined the doses of toxin that were most effective at reducing cortical and hippocampal ChAT activity without resulting in excessive mortality, we administered the toxin and examined the effects of selective cholinergic loss on spatial learning. We made bilateral injections of saline or immunotoxin $(1.8$ or $3.6 \mu \mathrm{g})$, believing that bilateral injections would distribute the toxin better than a unilateral injection (experiment 2) in the small mouse ventricles. Behavior was assessed using a neurological battery (Paul et al., 1997) and a $1 \mathrm{~d}$ version of the Morris water maze (Frick et al., 2000). The $1 \mathrm{~d}$ water maze task is sensitive to variations across the estrous cycle (Frick and Berger-Sweeney, 2001) and to species differences (Frick et al., 2000). At the end of behavioral testing, the ChAT and GAD activities of some of the mice were measured in the hippocampus, neocortex, and striatum to examine the specificity of the lesion effects. Cholinergic neurons in the striatum do not express p75 receptors in adulthood (Gage et al., 1989) and serve as a marker of nonspecific damage. In other mice, ChAT and p75 immunofluorescent staining was used to assess the colocalization of the two markers in the basal forebrain. p75 and calbindin staining in the cerebellum as well as calbindin and parvalbumin staining in the basal forebrain were assessed to determine the selectivity of the effects of the toxin.

\section{MATERIALS AND METHODS}

\section{Experiment 1}

Antibodies and cells. The anti-murine-p75 antibody (Advanced Targeting Systems) used in this work is described by Rao and Anderson (1997). It is a rat monoclonal antibody to the extracellular domain of murine p75 (Huber and Chao, 1995). 192 IgG has been described previously (Chandler et al., 1984). FITC-labeled goat anti-murine and anti-rat IgGs were obtained from Chemicon International (Temecula, CA). C6, a rat glioma cell line, was obtained from the American Type Culture Collection (ATCC) (Manassas, VA). NG3 cells, a subclone of NG108-15 cells, were also obtained from ATCC. Phenazine methosulfate (PMS) and (3-(4,5dimethylthiazol-2-yl)-5-(3-carboxymethoxyphenyl)-2-(4-sulfophenyl)-2Htetrazolium, inner salt (MTS) were obtained from Promega (Madison WI) and were used for cytotoxicity assays.

Synthesis of anti-murine-p75-SAP. The rat anti-murine-p75 antibody was chemically conjugated to saporin (Stirpe et al., 1983) as described previously (Wrenn et al., 1996). The molecule has $\sim 1.5 \mathrm{~mol}$ of saporin per mole of antibody.

Cytotoxicity assays. Cytotoxicity assays were performed as described previously (Kohls and Lappi, 2000). Briefly, cells were plated in wells of a 96-well plate and allowed to attach overnight. Samples were added at the indicated concentrations and incubated for $72 \mathrm{hr}$ (for NG3 cells) or $56 \mathrm{hr}$ (for C6 cells). PMS and MTS were added according to the manufacturer's instructions. Plates were read at $492 \mathrm{~nm}$ with a Molecular Dynamics SpectraMax 300 plate reader with SoftmaxPro software (Molecular Dynamics, Sunnyvale, CA) to quantitate the amount of formazan produced from MTS by cellular bioreduction. Data were analyzed using GraphPad Prism software (GraphPad, San Diego, CA).

Flow cytometric analysis. Studies were performed at Cytometry Research (San Diego, CA) on a FACScan flow cytometer (Becton Dickinson, San Jose, CA) with Lysys II or CellQuest software. Fluorescence was produced with an argon ion laser (488 nm excitation). Fluorescence emission was measured using a 530/30 filter (total events, 10,000 per sample). Cells were incubated with primary antibody, washed, and incubated with FITC-labeled secondary antibody.

\section{Experiment 2}

Animals. Fifty-four C57BL/6 (female and male) mice, 8-10 weeks of age at the beginning of the experiment, were used. The mice were housed by sex in groups of four to five on a $12 \mathrm{hr}$ light/dark cycle with food and water available ad libitum. All behavioral testing was conducted during the light cycle.

Surgery. All surgical procedures were conducted under aseptic conditions. Mice were weighed and anesthetized with $1.2 \%$ avertin $(0.2 \mathrm{ml} / 10$ gm body weight, i.p.). The anesthetized mouse was placed in the stereotaxic apparatus, a hole was drilled into the skull, and a syringe filled with either saline or toxin (of varying concentrations) was lowered stereotaxically into the right lateral ventricle at the following stereotaxic coordinates: anteroposterior, $-0.6 \mathrm{~mm}$; mediolateral, $+1.0 \mathrm{~mm}$ relative to the skull surface at bregma; and dorsoventral, $-2.2 \mathrm{~mm}$ relative to the dura at the injection site. A total of $0.5-1.0 \mu \mathrm{l}$ was injected over $5 \mathrm{~min}$, and the needle was left in place for an additional $5 \mathrm{~min}$. After surgery, survival rates, general health, and motility were monitored. Mice were killed for neurochemistry or histology 10-12 d after surgery, unless otherwise noted in Results.

$C h A T$ radioenzymatic assay. The mice $(n=40$ with varying doses of toxin; $n=10$ controls) were sedated with $\mathrm{CO}_{2}$ (Berger-Sweeney et al., 1994a) and decapitated. The frontoparietal cortex and hippocampus were dissected, weighed, frozen on dry ice, and stored at $-70^{\circ} \mathrm{C}$ until the assay. Using the method of Fonnum (1975), ChAT activity was determined by measuring the radiolabeled acetylcholine produced in brain homogenates from $\left[{ }^{14} \mathrm{C}\right]$ acetyl coenzyme-A and choline, as described previously (Arters et al., 1998). The protein content of the brain homogenates was determined by a Bradford or BCA protein assay.

$G A D$ radioenzymatic assay. GAD assays were performed on the same homogenates used for the ChAT assays. The activity of the enzyme GAD, which synthesizes GABA, was determined from the radiolabeled $\mathrm{CO}_{2}$ produced by GAD from $\mathrm{L}-\left[1-{ }^{14} \mathrm{C}\right]$ glutamic acid $(40-60 \mathrm{mCi} / \mathrm{mmol}$; New England Nuclear, Boston, MA) as described previously (Frick and Berger-Sweeney, 2001), using a $\left[{ }^{14} \mathrm{C}\right] \mathrm{CO}_{2}$ trapping technique (O'Connor et al., 1988).

Immunoperoxidase staining. Mice ( $n=2$ controls; $n=2$ at $1.8 \mu \mathrm{g}$ of anti-murine-p75-SAP; $n=2$ at $3.6 \mu \mathrm{g}$ of anti-murine-p75-SAP) were killed by cervical dislocation and transcardially perfused in $4 \%$ paraformaldehyde in sodium phosphate buffer, $\mathrm{pH}$ 7.4. The brains were removed, post-fixed with the perfusant for $2 \mathrm{hr}$, weighed, cryoprotected in $10 \%$ DMSO in $0.1 \mathrm{M}$ PBS. Next, sections were serially frozen at $60 \mu \mathrm{m}$, stored in 24 well tissue-collection clusters, and stained for choline acetyltransferase (AB144P goat anti-ChAT; Chemicon) (dilution 1:250) or calbindin (AB1778 rabbit anti-calbindin D-28K; Chemicon) (dilution $1: 2500)$.

Briefly, sections were rinsed three times (all washes 5 min each) in 0.1 M PBS and blocked for 60 min with $3 \%$ appropriate normal serum before overnight ( $48 \mathrm{hr}$ for calbindin) incubation at $4^{\circ} \mathrm{C}$ with a solution of primary antibody diluted in the above blocking solution. Sections were subsequently rinsed with PBS three times, incubated for $100 \mathrm{~min}$ in secondary antiserum (biotinylated rabbit anti-goat $\mathrm{IgG}$ or biotinylated goat anti-rabbit IgG; Vector Laboratories, Burlingame, CA), diluted 1:500 in PBS, and washed with PBS three times before incubation for 45 min in avidin-biotin-peroxidase complex in PBS (Vectastain; Vector Laboratories). Finally, sections were rinsed with PBS one time and with Tris $(100 \mathrm{~mm}, \mathrm{pH} 7.6)$ two times and incubated for $\sim 9 \mathrm{~min}$ in diaminobenzidine (DAB) tetrahydrochloride and $0.3 \% \mathrm{H}_{2} \mathrm{O}_{2}$ (according to the instructions provided with the Vector Laboratories DAB kit). Cobaltnickel enhancement was used with ChAT staining but not with calbindin staining. The DAB development reaction was stopped by adding excess 
ice-cold Tris buffer and washing two times. Sections were mounted, air-dried, cleared in xylene, and coverslipped with distyrene, plasticizer, xylene (DPX) (BDH Chemicals, Poole, UK). Sections were photographed under bright-field illumination using a Leica microscope (Leica, Deerfield, IL).

\section{Experiment 3}

Animals. The subjects were 42 male C57BL/6 mice between the ages of $8-10$ weeks at the time of surgery. Housing procedures were identical to those described in experiment 2.

Surgery and neurochemistry. Procedures were as described in experiment 2, except that 15 mice received saline injections (controls) and 27 mice received bilateral anti-murine-p75-saporin lesions (anti-murinep75-SAP-lesioned; $n=17$ received $1.8 \mu \mathrm{g}$ in $0.5 \mu \mathrm{l}$ into each ventricle, total dose of $3.6 \mu \mathrm{g} ; n=10$ received $0.9 \mu \mathrm{g}$ in $0.5 \mu \mathrm{l}$ into each ventricle, total dose of $1.8 \mu \mathrm{g}$ ). Also, striatal samples were gathered and assessed for ChAT and GAD activity.

One day water maze task. Behavioral testing began 12-15 d after the surgery. A circular tank (103 cm diameter) was filled with water $(24 \pm$ $2^{\circ} \mathrm{C}$ ) and surrounded by various extramaze cues. Water maze testing followed the protocol described by Frick et al. (2000). First, the mice received a four trial shaping procedure to teach them to locate the platform. No data were collected during shaping. On the following day, each mouse was given 12 spatial training trials, organized into three blocks of 4 trials; each block was separated by $30 \mathrm{~min}$. A transparent lucite platform $(10 \times 10 \mathrm{~cm})$ was submerged beneath the surface of the water, in the northwest quadrant of the tank. The sequence of the four start positions (north, south, east, and west) varied for each trial. Each animal was given $60 \mathrm{sec}$ to reach to the platform, on which it remained for $10 \mathrm{sec}$. If the platform was not located within $60 \mathrm{sec}$, the mouse was placed on it by the experimenter. The next trial started immediately after removal from the platform. After completion of the fourth trial of the block, the animal was placed in its home cage for $30 \mathrm{~min}$. Swim time (in seconds), path length (in centimeters), and swim speed (in centimeters per second) were recorded. One probe trial was conducted 30 min after completion of the spatial task. During this trial, the platform was collapsed, remaining unavailable for escape for $30 \mathrm{sec}$. The platform was then raised and was available for escape for an additional $30 \mathrm{sec}$. During the first $30 \mathrm{sec}$ of the probe trial, quadrant time (the percentage of time spent in the training quadrant) and proximity (average distance in centimeters to the platform; distances sampled 10 times/sec) were recorded. A cued task to test nonspatial reference memory was conducted 20 min after completion of the probe trial. A visible platform (covered with yellow tape) extended just above the water level and had a plastic circle $(8 \mathrm{~cm}$ in diameter, $0.5 \mathrm{~cm}$ thick) attached perpendicularly to it. The platform was moved to a different quadrant for each of the four trials.

The spatial training trial and cued task measures were averaged within a group for each block of four trials (spatial task) or a single trial (cued task) and analyzed using a one-way repeated-measures ANOVA. Oneway ANOVAs without repeated measures were performed on the probe trial measures.

Immunofluorescence staining. Perfusion and fixation of the tissue proceeded as described in experiment 2. Free-floating $50 \mu \mathrm{m}$ sections were incubated in 5\% normal donkey serum (NDS) and $0.2 \%$ Triton in PBS for $1 \mathrm{hr}$ at room temperature. Next the sections were incubated for $24 \mathrm{hr}$ at room temperature in a solution containing $2 \%$ NDS, $0.2 \%$ Triton in PBS, the primary antibody rabbit anti-p75 (1:400; Chemicon), and the primary antibody goat anti-ChAT (1:100; Chemicon). The sections were then incubated in both secondary antibodies for $1 \mathrm{hr}$ at room temperature. This solution contained donkey anti-rabbit (Cy3; Jackson ImmunoResearch, West Grove, PA) (1:100) and donkey anti-goat (Alexa 488; Molecular Probes, Eugene, OR) (1:100) as well as 0.2\% Triton in PBS. Sections were mounted on Superfrost Plus slides (VWR Scientific, West Chester, PA) using $90 \%$ glycerol in PBS solution. Other sections were stained for rabbit anti-calbindin (1:100; Chemicon) for $24 \mathrm{hr}$ at room temperature or for rabbit anti-parvalbumin (1:500; Swant, Bellinzona, Switzerland) for $24 \mathrm{hr}$ at $4^{\circ} \mathrm{C}$. These sections were blocked using $5 \%$ normal goat serum. For both calbindin and parvalbumin staining, the secondary antibodies were goat anti-rabbit (Alexa 594; Molecular Probes) (1:100). No cross-reactions among any of the secondary antibodies were noted. The fluorescent sections were analyzed using a Leica TCS confocal system. Images of the MS, nBM, or cerebellum were taken throughout the entire thickness of the $\approx 40 \mu \mathrm{m}$ section at $2 \mu \mathrm{m}$ intervals. The projected images were then fused for the photographs.
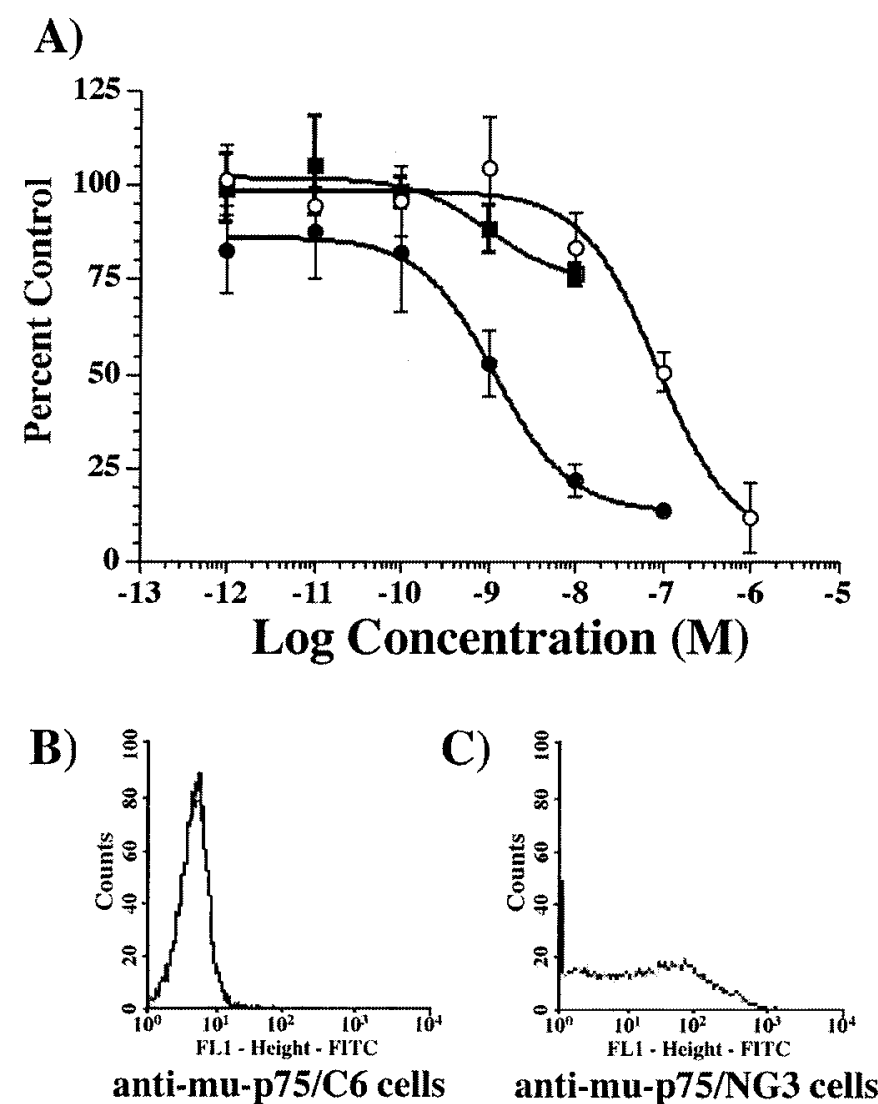

Figure 1. A, Cytotoxicity of anti-murine-p75-SAP to NG3 cells that express murine p75 and C6 glioma cells that do not. Anti-murine-p75SAP versus NG3 cells; $\bigcirc$, SAP versus NG3 cells; $\mathbf{\square}$, anti-murine-p75 versus C6 cells. $B$, FACS analysis of rat C6 glioma cells with anti-murinep75-SAP and FITC-labeled goat anti-rat IgG. This profile was identical to the profile of FITC-labeled secondary antibody alone. $C$, Labeling of NG3 anti-murine-p75 antibody and FITC-labeled goat anti-rat secondary antibody. Data show the binding of the antibody to the surface of cells that express murine $\mathrm{p} 75$.

\section{RESULTS}

\section{Experiment 1}

Anti-murine-p75-SAP is cytotoxic to cultured cells expressing murine p75. Cells in culture that express murine p75 (NG3 cells) were challenged with the anti-murine-p75-SAP immunotoxin (Fig. $1 A$ ). The immunotoxin is more toxic $\left(\mathrm{ED}_{50}=1.2 \times 10^{-9} \mathrm{M}\right)$ to these cells by a factor of almost 100 -fold over nontargeted saporin $\left(\mathrm{ED}_{50}=8.8 \times 10^{-8} \mathrm{M}\right)$. When tested against cells that do not express murine p75 (rat C6 glioma cells) (Zanellato et al., 1994), there is no significant difference between the effect of the immunotoxin and the effect of saporin, which enters by bulk phase endocytosis. These data are consistent with fluorescenceactivated cell sorter (FACS) analysis of these cells and the relevant antibodies. The anti-murine-p75 antibody recognizes NG3 cells but does not recognize C6 cells (Fig. 1,B,C).

\section{Experiment 2}

Our previous studies have shown that control and/or vehicle injections of uncoupled saporin did not produce neurotoxicity. The present study contained groups of mice with different doses of anti-murine-p75 SAP or saline injected intracerebroventricularly. 


\section{Intracerebroventricular immunotoxin doses of $7.1 \mu \mathrm{g} / \mu \mathrm{l}$ were lethal}

At the highest dose of toxin $(7.1 \mu \mathrm{g})$ five of seven mice died within $7 \mathrm{~d}$ of the toxin injections. The two surviving mice were killed at $7 \mathrm{~d}$ for neurochemistry because they had lost a significant amount of weight and their death appeared imminent. As such, the $7.1 \mu \mathrm{g}$ dose resulted in effectively a $0 \%$ survival rate. At the next highest dose $(3.6 \mu \mathrm{g}) 13$ of 19 mice survived, a $68 \%$ survival rate. At the $1.8 \mu \mathrm{g}$ dose, 10 of 13 mice survived, a $77 \%$ survival rate. All mice that did not survive began to exhibit severe weight loss within 3-7 $\mathrm{d}$ after the toxin injections. None of the mice that survived exhibited gross abnormalities in motility, such as dragging hindlimbs, as has been noted previously after intracerebroventricular injections of 192-SAP in rats (Berger-Sweeney et al., 1994b). Some of the surviving lesioned mice did appear jumpy and more agitated than controls $7 \mathrm{~d}$ after surgery, similar to rats with intracerebroventricular injections of 192-SAP (Berger-Sweeney et al., 1994b).

The $3.6 \mu \mathrm{g} / \mu \mathrm{l}$ dose was the most effective at reducing cholinergic markers in basal forebrain targets while sparing noncholinergic markers

Analysis of the dose-response data was performed by determining whether the mean depletion at each dose differed significantly from zero. This analysis revealed a significant depletion of hippocampal ChAT activity (Fig. $2 A$ ) at the $1.8 \mu \mathrm{g}\left(t_{(7)}=2.42 ; p=\right.$ $0.046)$ and $3.6 \mu \mathrm{g}\left(t_{(10)}=2.87 ; p=0.017\right)$ doses and a significant depletion of cortical ChAT activity (Fig. $2 B$ ) at the $3.6 \mu \mathrm{g}$ dose $\left(t_{(10)}=4.17 ; p=0.002\right)$. Depletion of cortical ChAT activity at the $1.8 \mu \mathrm{g}$ dose approached significance $\left(t_{(7)}=2.17 ; p=0.067\right)$. Although the depletion of ChAT activity in both areas was larger in magnitude at the $7.1 \mu \mathrm{g}$ dose, these comparisons did not reach significance because neurochemical data were only available for two of the seven mice injected at this dose. Depletion of hippocampal and cortical GAD activity (Fig. $2 C, D$ ) was not significant at any dose of the toxin ( $p$ values of $>0.15$ ); numerical differences were apparent in the $7.1 \mu \mathrm{g}$ group, although these differences were not statistically significant.

\section{Histological analyses confirmed a significant reduction in cholinergic markers but a sparing of noncholinergic markers in the basal forebrain}

ChAT-positive neurons throughout the MS (Fig. $3 A$ ), calbindinpositive neurons in the MS (Fig. 3D), and ChAT-positive neurons in the nBM (Fig. 3G) exhibited normal staining patterns after intracerebroventricular saline injections. The injection of the immunotoxin resulted in a dose-dependent decrease in ChATpositive neurons in the MS (Fig. $3 B, C$ ). However, the pattern of calbindin-positive neurons in the MS was unaltered by the toxin injections (Fig. 3E,F). ChAT-positive neurons in the nBM were not altered significantly by the immunotoxin injections (Fig. $3 H, I)$. In addition, we did not observe alterations in ChATpositive neurons throughout the striatum in the immunotoxin lesioned brains (data not shown).

\section{Experiment 3}

The general health of the anti-murine-p75-SAP-lesioned mice was slightly poorer than that of controls; however, motility and reflexes were normal

None of the mice in this study, either saline- or immunotoxininjected, showed overt behavioral signs of seizure activity. The postoperative health of the anti-murine-p75-SAP-lesioned mice was poorer than that of saline-injected controls, as indicated by
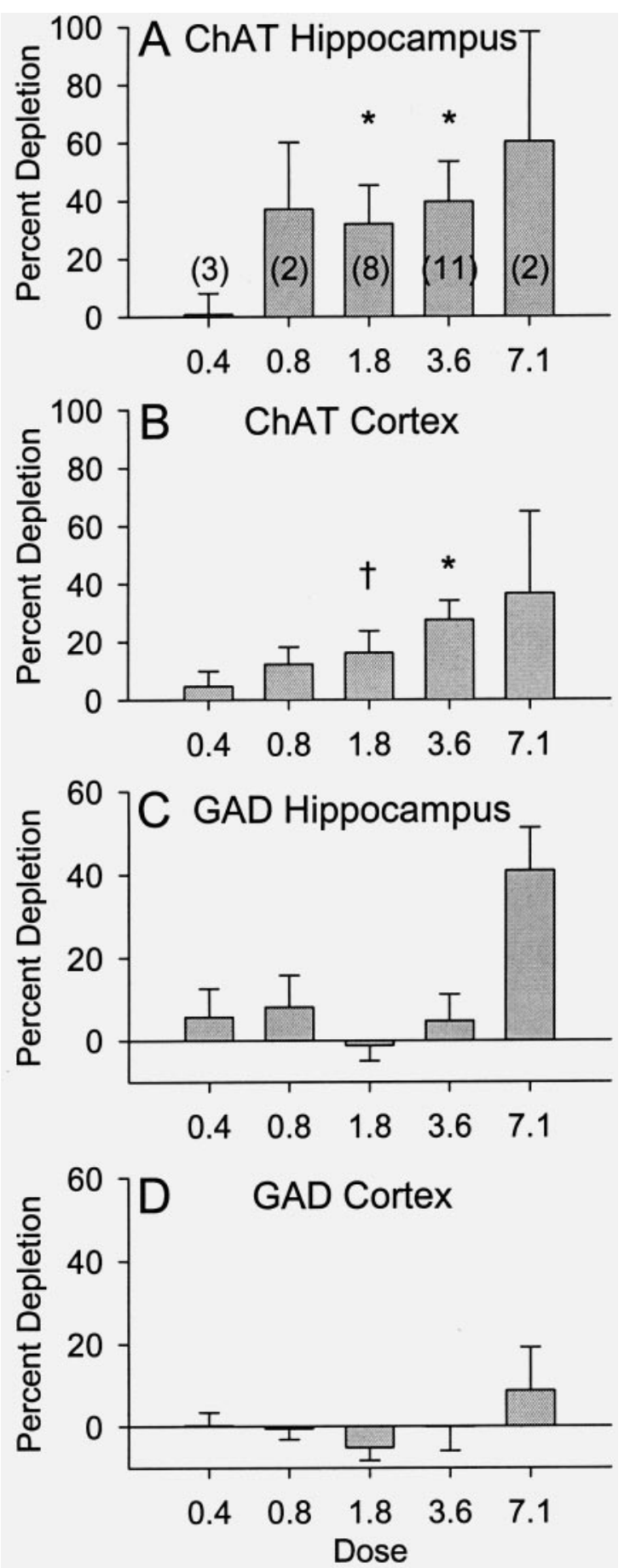

Figure 2. The percentage of depletion of ChAT $(A, B)$ and GAD $(C, D)$ after different doses (ranging from $0.4-7.1 \mu \mathrm{g} / \mu \mathrm{l}$ ) of intracerebroventricular injections of anti-murine-p75-SAP. Data are percentage of depletion \pm SEM. Anti-murine-p75-SAP injections result in a dose-dependent decrease in ChAT activity in the hippocampus $(A)$ and neocortex $(B)$. In contrast, there is not a dose-dependent loss of GAD activity in the hippocampus $(C)$ or neocortex $(D)$.

the consistently lower mean weights of the former group on days 2,6 , and 10 after surgery $\left(t_{(40)}<11.5 ; p<0.01\right)$. By day 10 , postsurgery saline-injected mice exhibited a mean weight of $24.6 \pm 0.5 \mathrm{gm}$, whereas lesioned mice exhibited a mean weight of $20.6 \pm 0.7 \mathrm{gm}$. Eight of the 27 anti-murine-p75-SAP-lesioned mice died between 4 and $7 \mathrm{~d}$ after surgery, despite hydrating 

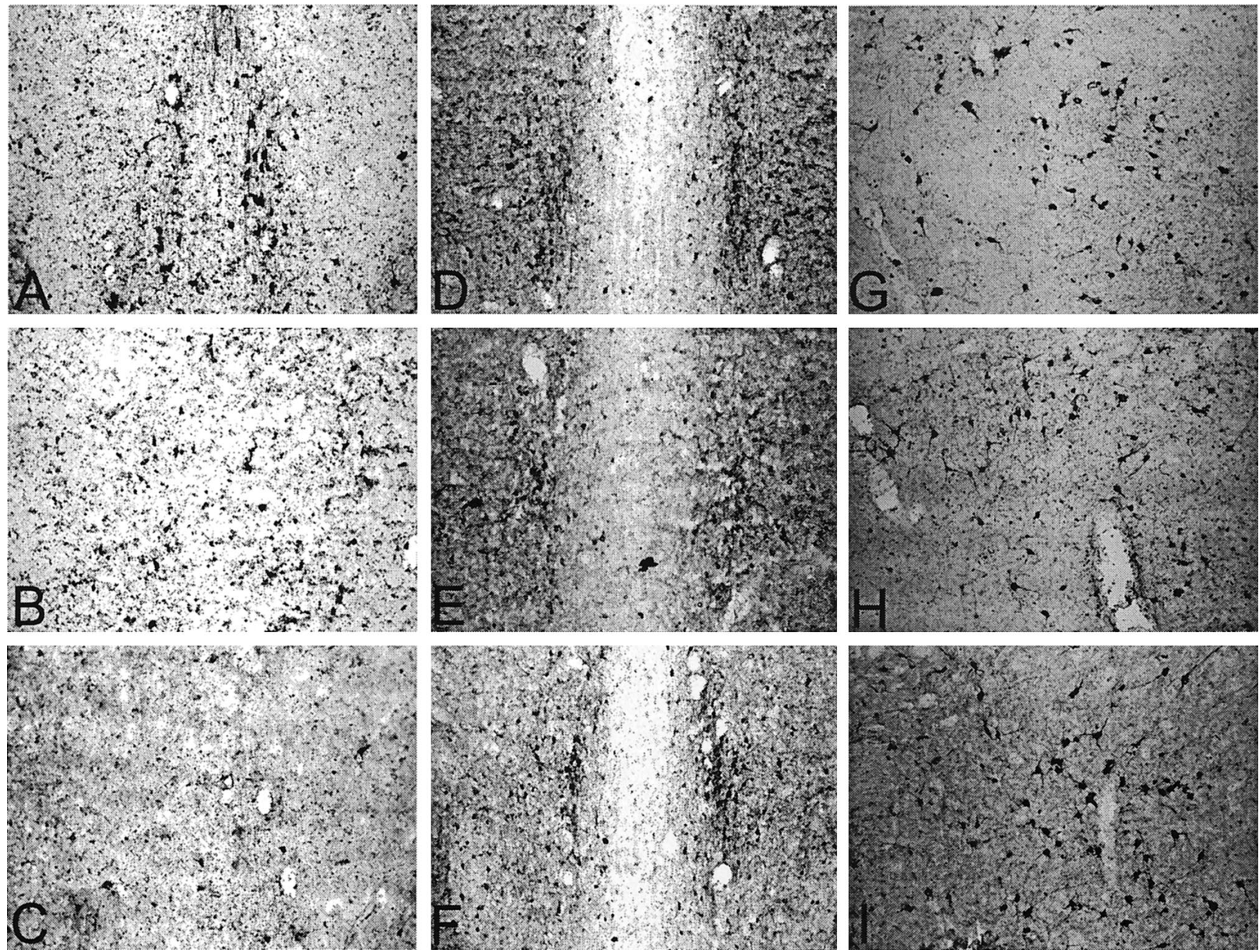

Figure 3. Immunohistochemistry for ChAT $(A-C, G-I)$ and calbindin $(D-F)$ after intracerebroventricular injection of saline $(A, D, G), 1.78 \mu \mathrm{g}$ of anti-murine-p75-SAP $(B, E, H)$, or $3.55 \mu \mathrm{g}$ of anti-murine-p75-saporin $(C, F, I)$. ChAT-positive neurons in the MS $(A-C)$ are lost dose-dependently, whereas calbindin-positive neurons in the MS $(D-F)$ are still present even after the highest dose of toxin. In contrast, there is no apparent loss of ChAT-positive neurons in the nBM at any dose of toxin $(G-I)$.

injections of $5 \%$ glucose in saline. Righting, placing, and grasping reflexes were normal in all of the control and SAP-lesioned mice that survived $7 \mathrm{~d}$ after surgery.

\section{Neurochemical results confirmed significant loss of ChAT} activity in basal forebrain targets of the anti-murine-p75-SAPlesioned mice who were tested behaviorally

Bilateral immunotoxin injections in this group of behaviorally tested mice resulted in a significant $(57.9 \%)$ depletion of ChAT $\left.t_{(20)}=-9.31 ; p<0.0005\right)$ in the hippocampus and in a significant $(19.3 \%)$ depletion of ChAT in the neocortex $\left(t_{(20)}=-2.48\right.$; $p=0.022)$ relative to controls. However, ChAT activity in the striatum was unaltered. In addition, GAD activity was unaltered in the hippocampus and striatum. However, neocortical GAD activity increased slightly $(8.7 \%)$ but significantly $\left(_{(20)}=3.28\right.$; $p=0.004)$.

Histological results confirmed the selectivity and specificity of the anti-murine-p75-SAP in the behaviorally tested mice

Saline-injected controls exhibited normal patterns of ChATpositive (Fig. 4A) and p75-positive (Fig. $4 B$ ) neurons throughout the extent of the MS. We observed a complete colocalization of
ChAT and p75 markers in the neurons of the MS (Fig. 4C). In addition, the saline-injected controls exhibited normal patterns of parvalbumin-positive (Fig. 4D) and calbindin-positive (Fig. 4E) neurons in the septal area. The bilateral ventricular anti-murinep75-SAP injections resulted in a dramatic loss of ChAT (Fig. $4 F, H$ ) and p75 (Fig. 4G,H) staining in the MS. However, noncholinergic calbindin-positive (Fig. $4 I$ ) and parvalbumin-positive (Fig. $4 J$ ) neurons exhibited staining patterns similar to controls.

In saline-injected controls, ChAT-positive (Fig. $5 A$ ) and p75positive (Fig. $5 B$ ) neurons exhibited normal staining patterns throughout the rostral/caudal extent of the substantia innomata/ nucleus basalis region. There was not complete colocalization of ChAT and p75 makers in the nBM. All p75-positive neurons observed were ChAT-positive; however, there were clusters of ChAT-positive neurons that did not contain p75 (Fig. 5C). The immunotoxin injections did not result in a dramatic loss of ChAT (Fig. $5 F, H$ ) or p75 (Fig. 5G,H). Calbindin (Fig. 5, $D$ vs $I$ ) and parvalbumin (Fig. 5, $E$ vs $J$ ) staining in the $\mathrm{nBM}$ was also undisturbed after the immunotoxin injections.

In the striatum, ChAT staining was virtually identical in control and lesioned mice (data not shown). In the cerebellum, p75 (Fig. 

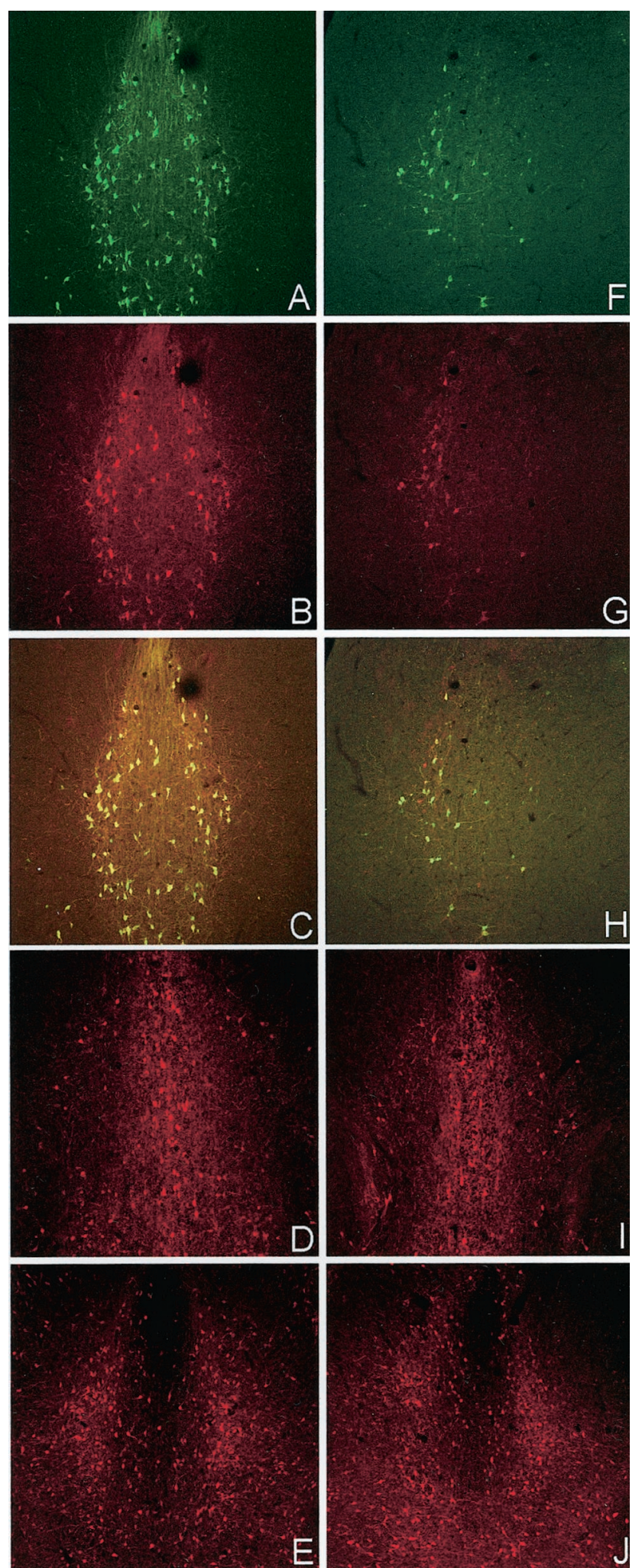

Figure 4. Immunofluorescence staining in the MS for $\operatorname{ChAT}(A, F), \mathrm{p} 75$ $(B, G)$, and double-labeling of ChAT/p75 $(C, H)$ after intracerebroventricular injections of saline $(A-C)$ or anti-murine-p75-SAP $(F-H)$. Immunofluorescence staining for noncholinergic neurons using calbindin $(D, I)$ and parvalbumin $(E, J)$ after intracerebroventricular injections of saline $(D, E)$ or anti-murine-p75-SAP $(I, J)$ is shown. ChAT-positive and p75-positive neurons in the MS are lost after immunotoxin injections, whereas there is no apparent loss of noncholinergic calbindin and parvalbumin staining in the septum.
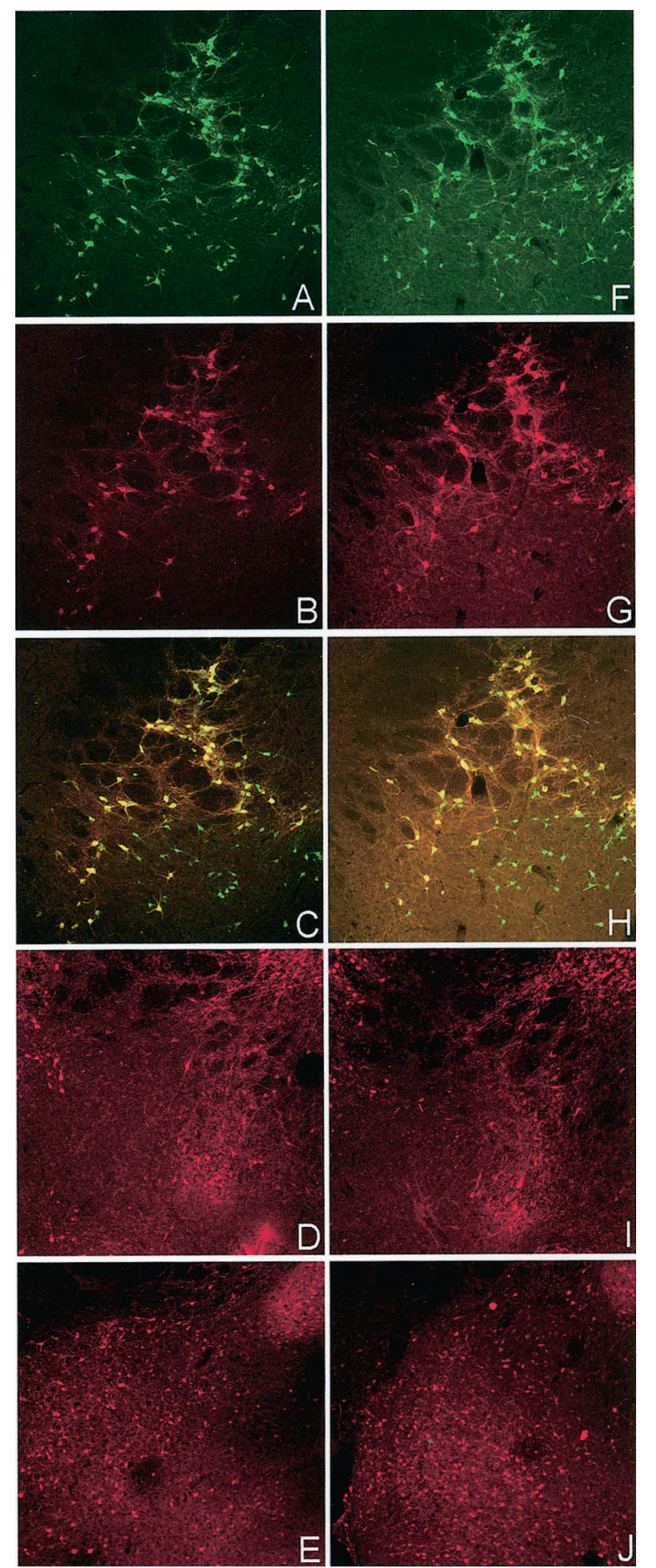

Figure 5. Immunofluorescence staining in the nBM for ChAT $(A, F)$, p75 $(B, G)$, and double-labeling of ChAT/p75 $(C, H)$ after intracerebroventricular injections of saline $(A-C)$ or anti-murine-p75-SAP $(F-H)$. Immunofluorescence staining for noncholinergic neurons using calbindin $(D, I)$ and parvalbumin $(E, J)$ after intracerebroventricular injections of saline $(D, E)$ or anti-murine-p75-SAP $(I, J)$ is shown. The immunotoxin injections do not have a dramatic effect on any of the markers in the nBM. 

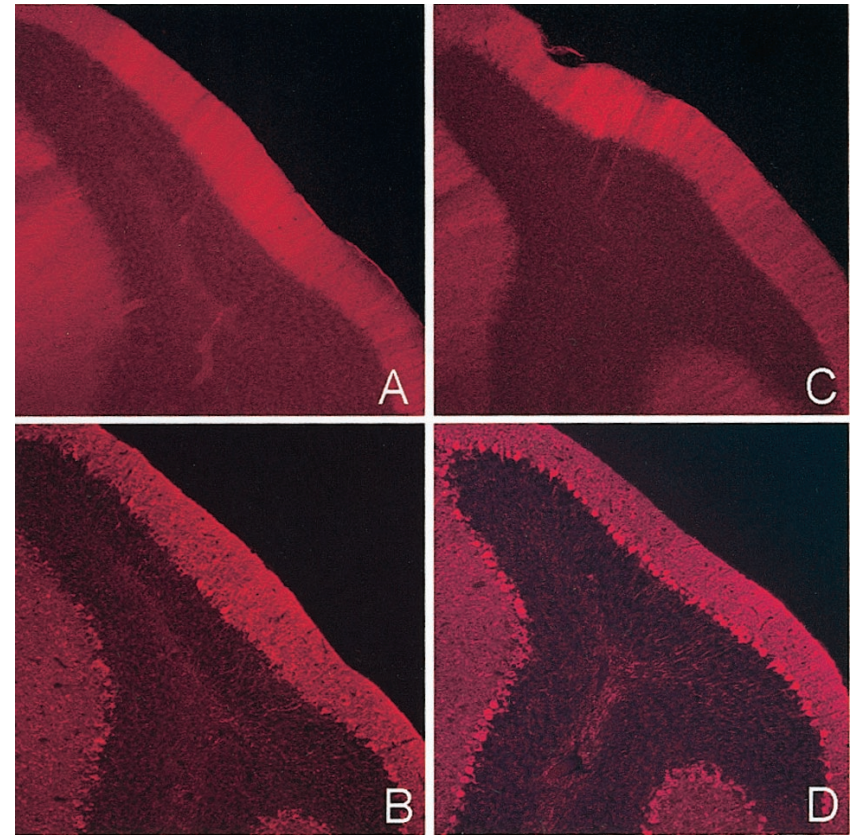

Figure 6. Immunofluorescence staining in the cerebellum of p75 $(A, C)$ and calbindin $(B, D)$ after intracerebroventricular injections of saline $(A$, $B)$ and immunotoxin $(C, D)$. The immunotoxin injections do not have a dramatic effect on any of these markers in the cerebellum.

$6 A, C$ ) and calbindin (Fig. $6 B, D$ ) staining were virtually identical in the saline- and immunotoxin-injected mice, with both exhibiting a strong but diffuse and somewhat irregular staining pattern. The striped banding patterns of p75 noted in rats (Heckers et al., 1994) were not readily visible in mice.

Anti-murine-p75-SAP-lesioned mice performed more poorly on all aspects of the water maze compared with controls

We first conducted group analyses comparing control mice $(n=$ 12) with mice with a $50-75 \%$ depletion of hippocampal ChAT activity but a $<20 \%$ depletion of hippocampal GAD activity $(n=$ 7). The ChAT threshold was chosen based on values in similar studies in rats; the GAD threshold was chosen because of a desire for minimal nonspecific damage (Waite et al., 1995; Wrenn et al., 1999). Analysis of water maze performance revealed main effects of the lesion on swim time on spatial training trials (Fig. 7A) $\left(F_{(1,17)}=15.75 ; p=0.001\right)$, on swim speed during training trials (Table 1) $\left(F_{(1,17)}=9.79 ; p=0.006\right)$, and on swim time on cued trials (Fig. $7 B)\left(F_{(1,17)}=7.29 ; p=0.015\right)$. An effect on platform crossings during probe trials approached significance $\left(F_{(1,17)}=\right.$ $3.00 ; p=0.10)$. These findings suggest some noncognitive impairment in the anti-murine-p75-SAP-lesioned mice. However, the main effect on swim time on spatial training trials remained when swim time on cued trials, a measure of motoric ability and motivation, was partialled out as a covariate $\left(F_{(1,16)}=5.92 ; p=\right.$ $0.027)$, suggesting that this effect on spatial training trials cannot be entirely accounted for by noncognitive factors. Additional measures of water maze performance are presented in Table 1, including thigmotaxis on the initial block of training trials and time in each of the four quadrants during the probe trial. No significant effects of the saporin lesion were observed on these measures.
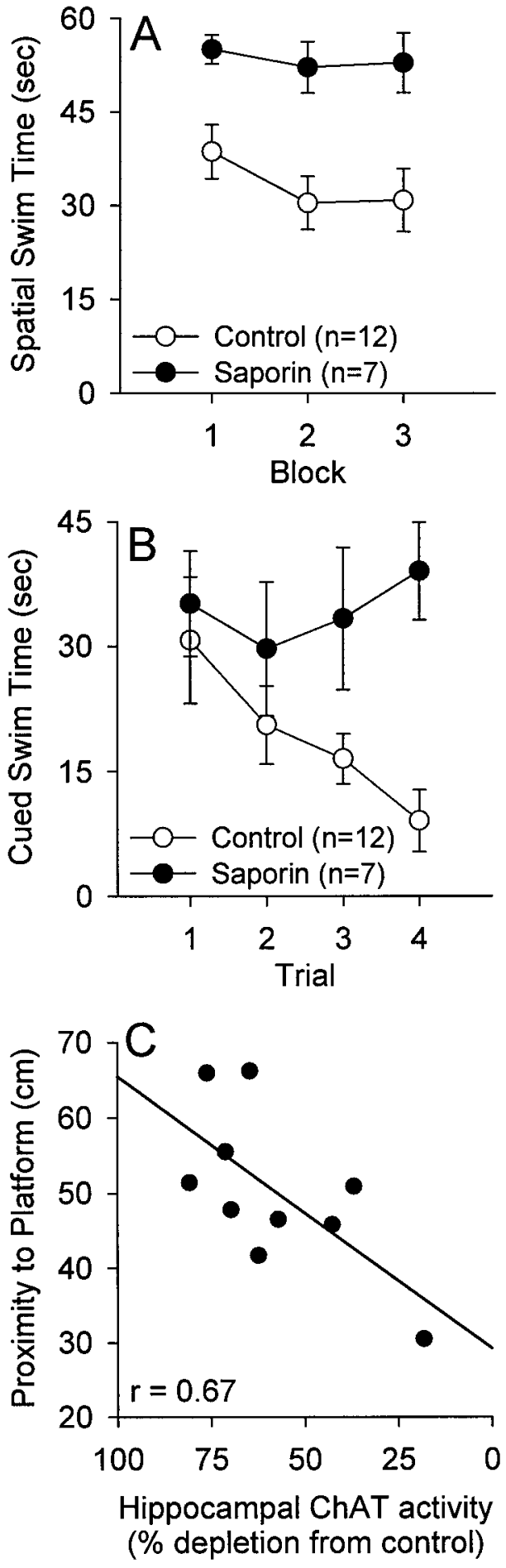

Figure 7. Swim maze performance in the saline-injected control mice and immunotoxin-injected mice. $A$, Time to reach the hidden platform on training trials was measured over three blocks in a $1 \mathrm{~d}$ swim maze task (spatial swim time). Control mice performed significantly better than lesioned mice on all three blocks of trials. $B$, Saporin-lesioned mice were also impaired in learning to swim to a visible platform, as measured by time to reach the visible platform on four training trials (cued swim time). However, the main effect on swim time on spatial training trials remained when swim time on cued trials was partialled out as a covariate. $C$, Correlation between proximity to platform on the spatial probe trial and hippocampal ChAT depletion. Better spatial performance was correlated significantly with higher hippocampal ChAT activity. 


\begin{tabular}{lrr}
\hline $\begin{array}{l}\text { Table 1. Additional measures of water maze performance } \\
\text { (mean } \pm \text { SEM) }\end{array}$ & \\
& $\begin{array}{l}\text { Control } \\
(n=12)\end{array}$ & \multicolumn{1}{c}{$\begin{array}{l}\text { Saporin } \\
(n=7)\end{array}$} \\
Measure & & \\
\hline Swim speed averaged across training & $14.46 \pm 1.08$ & $8.23 \pm 1.86^{*}$ \\
$\quad$ trial blocks $(\mathrm{cm} / \mathrm{sec})$ & $73.41 \pm 1.08$ & $79.41 \pm 3.46$ \\
Thigmotaxis during training block 1 $(\%)$ & & \\
Probe trial measures & $35.65 \pm 5.95$ & $31.0 \pm 6.62$ \\
$\quad$ Percentage of time in quadrant 1 & $33.25 \pm 7.62$ & $48.59 \pm 8.98$ \\
$\quad$ Percentage of time in quadrant 2 & $7.71 \pm 2.33$ & $9.07 \pm 3.43$ \\
$\quad$ Percentage of time in quadrant 3 & $23.36 \pm 5.28$ & $11.34 \pm 5.25$ \\
$\quad$ Percentage of time in quadrant 4 & $1.33 \pm 0.51$ & $0.14 \pm 0.14$ \\
Platform crossings & & \\
\hline
\end{tabular}

For the probe trial, quadrant 4 is the target quadrant (the one which contained the platform during training trials) and quadrant 2 is the opposite quadrant from the training quadrant. No significant differences were noted in thigmotaxis $(p=0.37)$ or on the probe trial measures (quadrants $1-3, p>0.22$; quadrant $4, p=0.15$; platform crossings, $p=0.10$ ). Mice showed an aversion to one of the quadrants of the swim pool, as has been noted previously in mice tested in this laboratory (Frick et al., 2000). Swim speed showed a significant lesion effect $(* p=0.006)$, as noted in Results.

\section{Hippocampal, but not neocortical ChAT activity, was significantly correlated with spatial learning ability}

To further explore the relationship between cholinergic loss and cognitive impairment, we considered the relationship between a loss of ChAT activity in the hippocampus and cortex and water maze performance in the group of anti-murine-p75-SAP-lesioned mice. For this analysis we included an additional three antimurine-p75-SAP-lesioned mice that had $<50 \%$ hippocampal ChAT depletion. This analysis revealed significant correlations between loss of hippocampal ChAT activity (percentage of decrease from control) and swim time on spatial training trials $(r=0.69 ; p=0.027)$, quadrant time on the probe trial $(r=-0.75$; $p=0.013)$, platform crossings on the probe trial $(r=-0.69 ; p=$ 0.028 ), and proximity to the platform on the probe trial (Fig. $7 C$ ) $(r=0.67 ; p=0.035)$. No correlations with cortical ChAT activity were significant, nor were there any significant correlations with cortical or hippocampal GAD activity. Partialling swim time on cued trials attenuated these correlations slightly (spatial swim time, $p r=0.55$; quadrant time, $p r=-0.66$; crossings, $p r=-0.56$; proximity, $p r=054)$; the correlation between a decrease in hippocampal ChAT activity and quadrant time still reached significance even when cued swim time was partialled out $(p=$ 0.053). Hence, there was evidence for a substantial relationship between hippocampal ChAT activity and spatial learning ability in anti-murine-p75-SAP-lesioned mice, even when possible motoric and motivational deficits were controlled statistically.

\section{DISCUSSION}

Our results suggest that we have created a toxin against mouse p75-bearing neurons that is both efficacious and selective in vivo. The in vitro data confirm the specificity of the toxin relative to unconjugated saporin, as well as its specificity for p75-bearing cells. The anti-murine-p75-SAP was no more toxic to cells that did not express the p75 receptor than was SAP alone. This selectivity was borne out in the in vivo studies: neurochemical analysis revealed a dose-dependent depletion of cortical and hippocampal ChAT activity after intracerebroventricular injection of the toxin. GAD activity, which is not expressed in cholinergic neurons and was used as a marker of nonspecific toxin effects, was altered only at the highest dose of toxin, one that proved lethal to the mice. Similarly, immunohistochemical anal- ysis indicated a selective loss of ChAT-immunopositive neurons, with preservation of noncholinergic neuronal populations in the basal forebrain (those expressing parvalbumin or calbindin).

There appear to be some important differences between the mouse anti-p75-SAP toxin and the previously characterized rat toxin, 192-SAP. First, the mouse toxin does not appear to have the same potency as the rat toxin. Intraventricular administration of 192-SAP $(2.7-4.0 \mu \mathrm{g})$ in the rat is capable of producing a $>90 \%$ depletion of cortical and hippocampal ChAT activity (Waite et al., 1995; Waite and Thal, 1996). In the mice used in experiment 3 , intracerebroventricular injections of anti-murinep75-SAP $(1.8-3.6 \mu \mathrm{g})$ produced a hippocampal and cortical ChAT depletion averaging 58 and $19 \%$, respectively. Higher doses of toxin produced greater levels of ChAT depletion in experiment 2 but also appeared to result in nonselective damage, indexed by losses in GAD activity in the same regions, and in any case were lethal to the mice. The difference in in vivo toxicity between the rat and mouse toxin is consistent with in vitro results. In vitro, the $\mathrm{ED}_{50}$ for 192-SAP on C6 cells is $1.6 \times 10^{-11}$, which is $\sim 2$ orders of magnitude more potent than the murine immunotoxin. Although direct comparisons of immunotoxin potencies cannot be made because the cells lines are different (C6 vs NG3), these data suggest that the murine immunotoxin is less potent than the rat immunotoxin. Other reasons that the mouse toxin could be less potent than the rat toxin include differences in the rate of internalization of the $\mathrm{p} 75$ receptor in the mouse brain, the transport of the receptor-toxin complex, or the different rates of distribution throughout the ventricular system. This decreased potency of the mouse toxin relative to the rat toxin may account for the increased lethality of the former $(23 \%$ lethality at an intracerebroventricular dose of $1.8 \mu \mathrm{g}$ ) because of nonspecific saporin damage. Although the toxicity is relatively high in the present study, additional titration of the dose-response curve, multiple microinjections at lower doses, increased postoperative care of the lesioned animals, and the use of intraparenchymal injections will likely result in decreased morbidity and/or mortality and increased effectiveness of the toxin.

The toxin is also less effective on nBM cholinergic neurons than on MS cholinergic neurons. Although neurochemical assays detected small, statistically significant decreases in cortical ChAT activity, no differences were obvious with regard to the density of ChAT-positive neurons in the nBM using histological methods, in contrast to the MS, in which loss was evident. Other reports suggest that the extent of the lesion after intracerebroventricular injection of saporin immunotoxins is greatest in structures that are the most proximal to the lateral ventricles, in other words, the MS (Wrenn et al., 1999; Ferreira et al., 2001). Another possible explanation for the apparently reduced sensitivity of nBM cholinergic neurons to anti-murine-p75-SAP is suggested by our double-labeling experiments, which indicate a small population of $\mathrm{nBM}$ cholinergic neurons that are not $\mathrm{p} 75$-immunoreactive. This incomplete colocalization of ChAT and p75 in the nBM is consistent with other findings in the mouse (Rossner et al., 2000), rat (Heckers et al., 1994), and sheep (Ferreira et al., 2001). In the rat, these ChAT-positive, p75-negative nBM cholinergic neurons project to the amygdala (Heckers et al., 1994); the projection patterns of these neurons have not yet been investigated in other species. Again, future anatomical studies and experiments with intraparenchymal injections of anti-murine-p75-SAP will help resolve this issue.

We also noted strong behavioral effects of the immunotoxic lesions in a $1 \mathrm{~d}$ version of the Morris water maze task. Intraven- 
tricular injections of 192-SAP in rats also produce impairments in water maze performance (as well as other tasks), a result which is sometimes attributed to the cerebellar damage and motoric impairments that accompany such lesions (Wrenn and Wiley, 1998; Waite et al., 1999; for review, see Baxter and Chiba, 1999). It is interesting that we observed no qualitative differences in cerebellar p75 or calbindin staining in the anti-murine-p75-SAP-lesioned mice, as well as no gross motoric abnormalities, although there were alterations in swim speed and performance of the cued version of the water maze task. These findings suggest that cerebellar damage was considerably less (or nonexistent) than what is seen in rats after intracerebroventricular 192-SAP injections. Interestingly, intracerebroventricular immunotoxin injections in sheep reportedly do not result in cerebellar damage, despite the existence of p75-positive Purkinje cells (Ferreira et al., 2001). In the current study, we cannot exclude the possibility that the cerebellar damage in the mice was below our limit of detection using immunocytochemical methods.

We also observed significant correlations in the immunotoxin lesion group between hippocampal ChAT depletion and behavioral impairment in the water maze, effects that are not entirely accounted for by motoric impairments caused by the lesions. Therefore, the findings of the current study provide the first report of significantly impaired water maze performance, without concomitant cerebellar damage, after specific cholinergic basal forebrain lesions. In addition, these behavioral impairments were associated with a moderate cholinergic depletion (57\% in MS and $18 \%$ in $\mathrm{nBM}$ ). The current results appear to be in contrast to the many reports in rats that suggest that water maze performance is unimpaired after extensive lesions to the MS and nBM (for review, see Baxter and Chiba, 1999). There are several possibilities for the differences between previous studies in rats and the current study in mice. First, the current study uses a $1 \mathrm{~d}$ water maze task instead of a multiday water maze task. The $1 \mathrm{~d}$ format may rely more heavily on short-term or working memory processes than the longer multiday task. Perhaps the former type of memory is more susceptible to cholinergic damage. Second, there may be significant species differences on this task that confound the results (Frick et al., 2000). Third, the precise pattern of damage created by the rat and mouse toxin may differ. Despite these caveats, the current study suggests that the cholinergic basal forebrain is involved critically in short-term spatial learning in mice.

The potential applications of selective immunotoxins for mice are extensive. The explosion of research using genetically modified mice to determine the roles of particular genes in normal brain function, as well as to create models of human diseases, has required parallel research into the neural mechanisms of behavior in genetically normal mice to provide a basis for the interpretation of functional deficits after genetic manipulation. It is becoming more apparent that behavioral tasks developed in rats may be solved differently by mice and may involve different neural substrates (McNamara et al., 1996; Gerlai and Clayton, 1999; Frick et al., 2000). The ability to perform selective neurochemical lesions in mice such as those that have been done in rats (and in other species) will further extend the comparative analysis of brain mechanisms of behavior in mice.

Genetic modification of mice is also a time-consuming and expensive process. Interpretation of phenotypic changes after gene knockout can be complicated by genetic backgrounds and developmental expression of the gene. In addition, current knockout methods provide little anatomical specificity. Selective immunotoxins provide a means of creating a spatially and temporally restricted ablation of a neurochemically defined neuronal population in mice that is extremely cost- and time-effective relative to creation of a genetically modified mouse. Of course, this advantage must be weighed against the disadvantage of a partial rather than a complete loss of the neurotransmitter of interest, and the toxicity associated with this method.

Perhaps the most exciting application of the mouse anti-p75SAP toxin will be the combination of selective cholinergic lesions with genetic modification to explore the role of cholinergic damage in the development of neuropathology and neuropsychological impairment in mouse models of Alzheimer's disease. The mechanism of cholinergic neuron death in AD is still not well understood. It is also not known whether the loss of these neurons contributes to the progression of neuropathology in AD. Because a variety of mouse models of AD now exist that express key neuropathological and behavioral characteristics of the disorder, it will now be possible, using the anti-murine-p75-SAP toxin, to determine whether cholinergic neuron loss exacerbates pathology and behavioral impairment in these mice.

\section{REFERENCES}

Arters J, Hohmann CF, Mills J, Olaghere O, Berger-Sweeney J (1998) Sexually dimorphic responses to neonatal basal forebrain lesions in mice. I. Behavior and neurochemistry. J Neurobiol 37:582-594.

Baxter MG, Chiba AA (1999) Cognitive functions of the basal forebrain. Curr Opin Neurobiol 9:178-183.

Berger-Sweeney J, Berger UV, Sharma M, Paul CA (1994a) Effects of carbon dioxide-induced anesthesia on cholinergic parameters in rat brain. Lab Anim Sci 44:369-371.

Berger-Sweeney J, Heckers S, Mesulam MM, Wiley RG, Lappi DA, Sharma M (1994b) Differential effects on spatial navigation of immunotoxin-induced cholinergic lesions of the medial septal area and nucleus basalis magnocellularis. J Neurosci 14:4507-4519.

Bornemann K, Staufenbiel M (2000) Transgenic mouse models of Alzheimer's disease. Ann NY Acad Sci 908:260-266.

Chandler CE, Parsons LM, Hosang M, Shooter EM (1984) A monoclonal antibody modulates the interaction of nerve growth factor with PC12 cells. J Biol Chem 259:6882-6889.

Ferreira G, Meurisse M, Tillet Y, Levy F (2001) Distribution and colocalization of choline acetyltransferase and p75 neurotrophin receptors in the sheep basal forebrain: implications for the use of a specific cholinergic immunotoxin. Neuroscience 104:419-439.

Fonnum F (1975) A rapid radiochemical method for the determination of choline acetyltransferase. J Neurochem 24:407-409.

Freund TF (1989) GABAergic septohippocampal neurons contain parvalbumin. Brain Res 478:375-381.

Frick KM, Berger-Sweeney J (2001) Spatial reference memory and neocortical neurochemistry vary with the estrous cycle in C57BL/6 mice. Behav Neurosci 115:229-237.

Frick KM, Stillner ES, Berger-Sweeney J (2000) Mice are not little rats: species differences in a one-day water maze task. NeuroReport 11:3461-3465.

Gage FH, Batchelor P, Chen KS, Chin D, Higgins GA, Koh S, Deputy S, Rosenberg MB, Fischer W, Björklund A (1989) NGF receptor reexpression and NGF-mediated cholinergic neuronal hypertrophy in the damaged adult neostriatum. Neuron 2:1177-1184.

Gerlai R, Clayton NS (1999) Analysing hippocampal function in transgenic mice: an ethological perspective. Trends Neurosci 22:47-51.

Heckers S, Ohtake T, Wiley RG, Lappi DA, Guela C, Mesulam M (1994) Complete and selective cholinergic denervation of rat neocortex and hippocampus but not amygdala by an immunotoxin against the p75 NGF receptor. J Neurosci 14:1271-1289.

Huber LJ, Chao MV (1995) Mesenchymal and neuronal cell expression of the p75 neurotrophin receptor gene occur by different mechanisms. Dev Biol 167:227-238.

Janus C, Chrishti M, Westaway D (2000) Transgenic mouse models of Alzheimer's disease. Biochim Biophys Acta 1502:63-75.

Kasa P, Rakonczay Z, Gulya K (1997) The cholinergic system in Alzheimer's disease. Prog Neurobiol 52:511-535.

Kohls MS, Lappi DA (2000) Mab-SAP: a tool for evaluating antibody efficacy for use in an immunotoxin. Biotechniques 28:162-165.

McNamara RK, Namgung U, Routtenberg A (1996) Distinctions between hippocampus of mouse and rat: protein F1/GAP-43 gene expression, promoter activity, and spatial memory. Brain Res Mol Brain Res 40:177-187.

O'Connor LH, Nock. B, McEwen BS (1988) Regional specificity of 
$\gamma$-aminobutyric acid receptor regulation by estradiol. Neuroendocrinology 47:473-481.

Paul CA, Beltz BS, Berger-Sweeney J (1997) Discovering neurons: a compilation of classic and novel laboratory exercises in neuroscience. Cold Spring Harbor: Cold Spring Harbor Laboratory.

Price D, Wong P, Markowska A, Lee M, Thinakaren G, Cleveland D, Sisodia S, Borchelt D (2000) The value of transgenic models for the study of neurodegenerative diseases. Ann NY Acad Sci 920:179-191.

Rao MS, Anderson DJ (1997) Immortalization and controlled in vitro differentiation of murine multipotent neural crest stem cells. J Neurobiol 32:722-746.

Rossner S, Schliebs R, Bigl V (2000) Intracerebroventricular inf usion of $\mathrm{CHO} 5$, a rat monoclonal antibody directed against mouse low-affinity nerve growth factor receptor (p75NTR), specifically labels basal forebrain cholinergic neurons in mouse brain. Metab Brain Dis 15:17-27.

Stirpe F, Gasper-Campani A, Barbieri L, Falasca A, Abbondanza A, Stevens WA (1983) Ribosome-inactivating proteins from the seeds of Saponaria officinalis L. (soapwort) or Agrostemma githago L. (corn cockle) and of Asparagus officianlis (asparagus) and from the latex of Hura crepitans L. (sandbox tree). Biochem J 216:617-625.

Waite JD, Chen AD, Wardlow ML, Wiley RG, Lappi DA, Thal LJ (1995) 192 immunoglobulin G-saporin produces graded behavioral and biochemical changes accompanying the loss of cholinergic neurons of the basal forebrain and cerebellar Purkinje cells. Neuroscience 65:463-476.

Waite JJ, Thal LJ (1996) Lesions of the cholinergic nuclei in the rat basal forebrain: excitotoxins vs an immunotoxin. Life Sci 58:1947-1953.
Waite JJ, Wardlow ML, Power AE (1999) Deficit in selective and divided attention associated with cholinergic basal forebrain immunotoxic lesion produced by 192-saporin; motoric/sensory deficit associated with Purkinje cell immunotoxic lesion produced by OX7-saporin. Neurobiol Learn Mem 71:325-352.

Wenk G, Willard L (1998) The neural mechanisms underlying cholinergic cell death within the basal forebrain. Int $\mathrm{J}$ Dev Neurosci 16:729-735.

Whitehouse P (1998) The cholinergic deficit in Alzheimer's disease. J Clin Psychiatry 59:19-22.

Wiley RG (1992) Neural lesioning with ribosome-inactivating proteins: suicide transport and immunolesioning. Trends Neurosci 15:285-290.

Wrenn CC, Wiley RG (1998) The behavioral functions of the cholinergic basal forebrain: lessons from 192 IgG-saporin. Int J Dev Neurosci 16:595-602.

Wrenn CC, Picklo MJ, Lappi DA, Robertson DR, Wiley RG (1996) Central noradrenergic lesioning using anti-DBH-saporin: anatomical findings. Brain Res 740:175-186.

Wrenn CC, Lappi DA, Wiley RG (1999) Threshold relationship between lesion extent of the cholinergic basal forebrain in the rat and working memory impairment in the radial maze. Brain Res 847:284-298.

Zanellato A, Facci L, Petrelli L, Dal Toso R, Skaper SD (1994) Characterization of growth-dependent regulation of the nerve growth factor receptor gp140trk in rat C6 glioma cells. Brain Res Mol Brain Res 23:299-309. 